\title{
CT evaluation of accurate catheter tip positioning techniques in upper arm central venous port implantation: intracavitary ECG versus fluoroscopy
}

Weiwei Kong, Ling Yuan, Yang Chen The Comprehensive Cancer Centre of Drum Tower Hospital, Medical School of Nanjing University, Clinical Cancer Institute of Nanjing University, Jiangsu Province, China

\section{Fig.1 Illustration of measurement method}

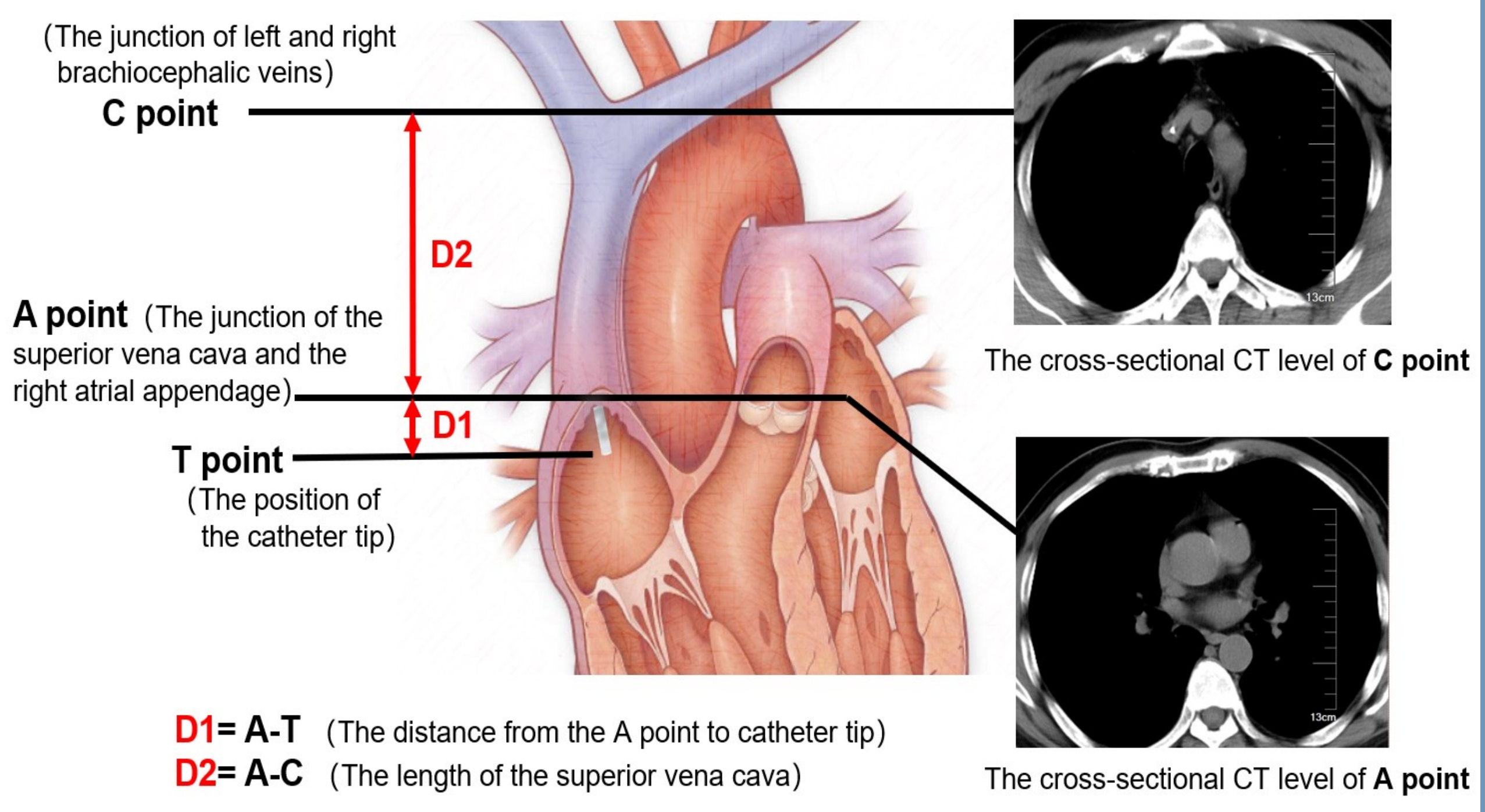

Fig. 2 Rate of Proper Tip Position in different groups

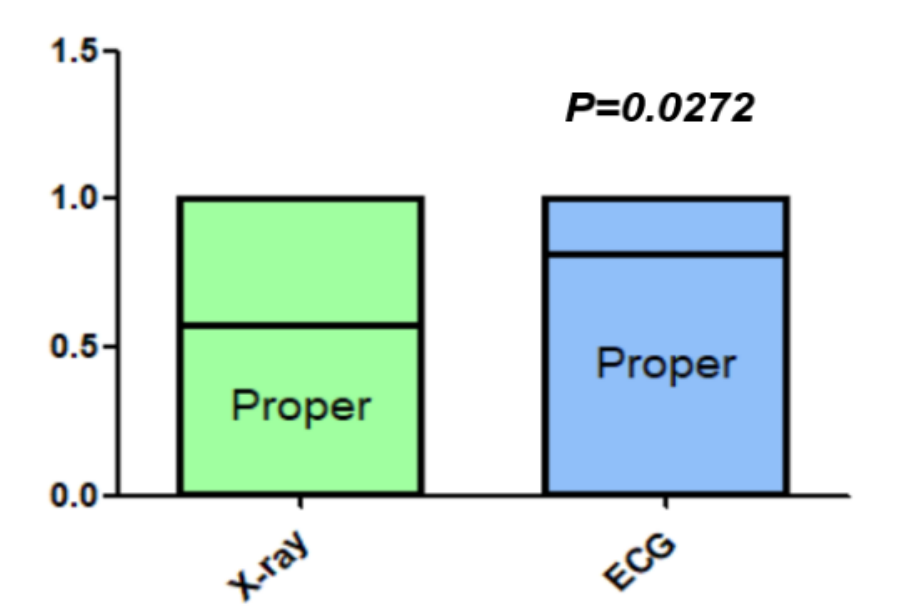

Fig. 3 D1 in different groups

(Tip Position superior to A point)

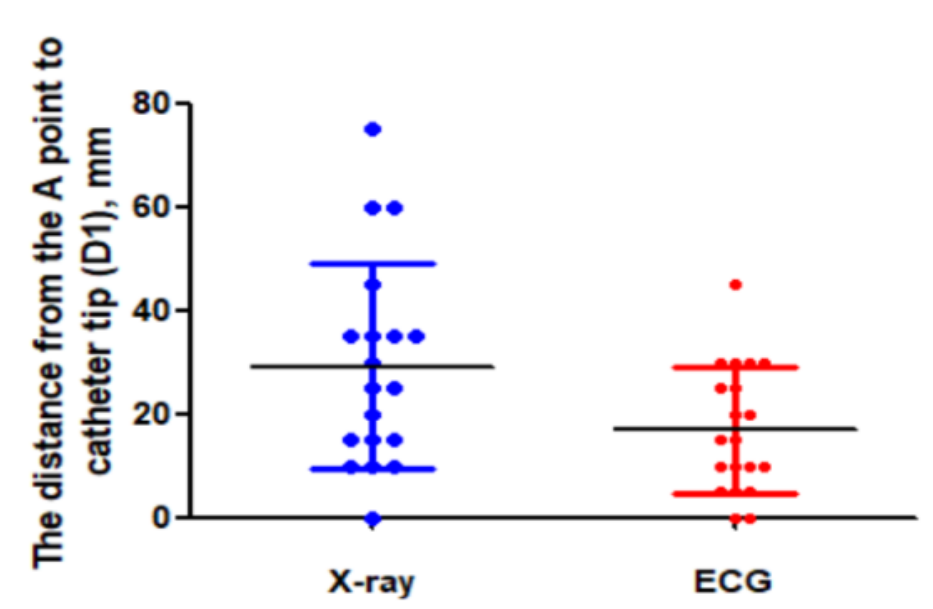

\section{Background}

Implantation of upper arm central venous port catheters require more accurate tip positioning techniques. Although chest X-ray radiographs are currently the "gold standard" for determining catheter tip location, intracavitary electrocardiogram (IC-ECG) -guided techniques are found to be more advantageous.

\section{Objective}

To analyze the location of the catheter tip by postoperative $\mathrm{CT}$ scan and compared two positioning techniques.

\section{Material and Methods}

85 patients received upper arm central venous port implantation from August 2014 to December 2017, and 55 patients who underwent follow-up CT scan were recruited. In IC-ECG group, the ideal end position for the catheter was where the ECG showed $\mathrm{P}$ wave at its maximum height, and the ECG was the only guidance method during operation. In $\mathrm{X}$-ray group, catheter tip location was confirmed by posterior/anterior (PA) chest X-ray film. The proper location of the catheter tip by CT scan was defined as in the lower third of the superior vena cava or within $2 \mathrm{~cm}$ below cavoatrial junction. The length of the superior vena cava and the distance from the catheter tip to the cavo-atrial junction were measured. ( Fig. 1)

\section{Results}

There were 28 patients in X-ray group, and 27 patients in ICECG group. In IC-ECG group, $81.48 \%$ of the catheter tip was located at proper position by CT scan, as compared to $57.14 \%$ of the tip position in $\mathrm{X}$-ray group was proper $(\mathrm{P}=0.027) .20$ patients in IC-ECG group and 19 in $\mathrm{X}$-ray group were confirmed their catheter tip being located in the superior vena cava, and average distance from the catheter tip to the cavo-atrial junction (D1) was $17 \pm 2.7 \mathrm{~mm}$ and $29 \pm 4.5 \mathrm{~mm}(\mathrm{P}=0.028)$ respectively. While 7 patients in ICECG group and 9 in X-ray group were confirmed their catheter tip being located below the cavo-atrial junction, and average distance from the catheter tip was $8.7 \pm 1.4 \mathrm{~mm}$ and $14 \pm 2.8 \mathrm{~mm}(\mathrm{P}=0.107)$ respectively. ( Fig. 2 and Fig. 3)

\section{Conclusion}

The accuracy of X-ray assessment of the catheter tip position was questioned by the CT scan, possibly due to the large variation in the length of the superior vena cava. Electrocardiographic guidance has been shown more effective than fluoroscopy to position the tip of central venous devices. It is necessary to conduct further randomized controlled studies to confirm these findings. 